\section{Impacto del Seguro Popular y de la disponibilidad de profesionales de salud en el control de la hipertensión arterial en México}

La hipertensión arterial (HTA) afecta a más de nueve millones de adultos en México y esta cifra debe aumentar a medida que aumentan las tasas de obesidad en la población. La HTA incrementa considerablemente el riesgo de enfermedades cardiovasculares, como la enfermedad isquémica y el infarto (segunda y tercera causas de muerte en México, respectivamente). El gasto esperado este año para tratar la HTA es de más de 275 millones de dólares estadounidenses. Se ha demostrado que la reducción de la HTA reduce significativamente la incidencia de enfermedades cardiovasculares y la mortalidad asociada. A pesar de disponer de medicamentos eficaces, el tratamiento de la HTA no es el óptimo y su eficacia varía de una región a otra del país.

La falta de seguro médico ha sido un obstáculo clave permanente para el tratamiento antihipertensivo y para el uso de los servicios de salud en general. El reciente programa Seguro Popular, que pretende extender legalmente el seguro de salud a todas las personas que no poseen un seguro médico en México para el año 2010 (alrededor de 50 millones de personas), puede mejorar el acceso al tratamiento de la HTA y, seguramente, los resultados sanitarios del país. El paquete asegurador cubre 249 intervenciones, entre ellas el diagnóstico y el tratamiento de la HTA. La ley, sin embargo, estipula que la prioridad en la afiliación la tienen los hogares pobres de zonas con grandes carencias económicas, los habitantes de zonas rurales y los grupos indígenas, así como las familias que tienen necesidades mayores de atención sanitaria. Por ende, la mayor parte de la población cubierta actualmente por este seguro pertenece a los grupos con ingresos más bajos. Además, el Seguro Popular se diseñó para que se comenzara a aplicar en las comunidades en las que existieran instalaciones de salud suficientemente equipadas para ofrecer los servicios contemplados en el paquete del seguro. En las zonas rurales y de bajos ingresos, la falta de suministros puede impedir la efectividad del programa con respecto al tratamiento antihipertensivo.

Para investigar si el Seguro Popular es suficiente por sí solo para aumentar la cobertura de tratamiento antihipertensivo en adultos de México o si su efecto es mayor en las zonas que cuentan con más recursos humanos, se examinaron las contribuciones independientes y combinadas del estatus de asegurado y de la cantidad de profesionales sanitarios.

Para ello se estudiaron 2967 adultos hipertensos de 20 años o más sin seguro médico, de ellos 1065 se parearon por edad con igual número de personas cubiertas por el Seguro Popular, por lo que el estudio abarcó a un total de 4032 personas. El número de médicos y enfermeros $(n=12566)$ se tomó del Registro Nacional de Infraestructura de Salud de México correspondiente a 2005.

Los resultados mostraron una asociación significativa entre estar cubierto por el Seguro Popular y una mayor tasa de tratamiento por HTA y de control de la tensión arterial. Además, el Seguro Popular fue más efectivo en las zonas que contaban con una mejor relación entre el número de profesionales y el de pacientes.

En 2005, casi la sexta parte de los adultos de México (cerca de cinco millones de personas) podían optar por el Seguro Popular. Se espera que para el año 2010, el Seguro Popular permita incrementar la asignación de recursos públicos para aumentar el pago de los servicios de salud de cada familia, de $\$ 268,00$ en 2001 (de un gasto medio por hogar de $\$ 4032,00$ ) a $\$ 677,00$. La prevención secundaria de las enfermedades cardiovasculares, como resultado de mayores tasas de tratamiento y de control de la HTA, puede compensar los gastos que ocasione la ampliación de la cobertura.

A diferencia de otros estudios, estos resultados no demostraron que la presencia de profesionales sanitarios tenga un efecto independiente en el tratamiento de la HTA. El hecho de haber encontrado una relación directa y significativa entre el estar cubierto por el Seguro Popular y la presencia de profesionales sanitarios con respecto a la cobertura del tratamiento antihipertensivo indica que el Seguro Popular no fue suficiente por sí solo. En cambio, se comprobó que se obtiene el mayor impacto de este seguro sobre la cobertura del tratamiento contra la HTA cuando la relación entre el número de médicos y de pacientes es alta.

En conclusión, en este trabajo se demuestra que el Seguro Popular está teniendo un impacto positivo en la cobertura del tratamiento antihipertensivo en México, especialmente en las zonas en que la concentración de profesionales sanitarios es alta. Además de hacer esfuerzos adicionales para comprender mejor los factores que inciden en la falta de 
control de la HTA en México, se debe ampliar la cobertura de los seguros de salud y aumentar la cantidad de médicos y enfermeros en las zonas en las que aún no son suficientes. El éxito del Seguro Popular debe constituir un ejemplo para otros países en desarrollo que aspiran a garantizar la atención médica a las personas que no cuentan con un seguro de salud. (Bleich SN, Cutler DM, Adams AS, Lozano R, Murray CJL. Impact of insurance and supply of health professionals on coverage of treatment for hypertension in Mexico: population based study. Br Med J. 2007;335:875.) 